\title{
RETOS ACTUALES Y FUTUROS EN IMPLANTES DE RODILLA Y CADERA EN MÉXICO
}

\author{
G.C. Mondragón-Rodríguez ${ }^{* 1}$, J.M. Alvarado-Orozco ${ }^{1}$, A. Gómez-Ortega ${ }^{1}$, N. Camacho*2. \\ 1: Dr, CIDESI - CONMAD, Ingeniería de Superficies y Manufactura Aditiva, Querétaro, Av. Pie de la \\ Cuesta 702, 76125 Querétaro, Oro. México.
}

2: Ph.D., CIDESI - CENTA, Centro Nacional de Tecnologías Aeronáuticas, Carretera Estatal 200, QuerétaroTequisquiapan Km 23 No. 2254, Colón, Querétaro, México.

*E-Mail: guillermo.mondragon@cidesi.edu.mx, nayeli.camacho@cidesi.edu.mx,

\begin{tabular}{ll}
\hline PALABRAS CLAVE & RESUMEN \\
\hline Artroplastia de rodilla & En este trabajo se realizó un análisis documental enriquecido por \\
Artroplastia de cadera & entrevistas con médicos especialistas acerca de la artroplastia de rodilla \\
Prótesis ortopédicas & y de cadera con el fin de identificar los avances más recientes en estos \\
Impresión 3D & tópicos, así como los retos y perspectivas futuras que se presentan tanto \\
Manufactura aditiva & en el ámbito quirúrgico como en el desarrollo de los reemplazos \\
Recubrimientos & articulares y sus materiales. México cuenta con una gran experiencia \\
Auto-lubricantes & clínica en la colocación de prótesis para reemplazo articular de rodilla, \\
& cadera, hombro, mano, y tobillo. Asimismo, la experiencia clínica en la \\
& colocación de implantes maxilo- y craneo-faciales es extensa. Sin \\
& embargo, el resultado del Simposio Nacional - Prótesis ortopédicas: \\
& Estatus actual en México (https://protesismexico.com/) mostró que hay \\
& una necesidad imperante de diseñar y fabricar implantes que se adapten \\
& a la población mexicana, buscando la personalización. Por su parte, en \\
& México, las investigaciones de los materiales para implantes \\
& ortopédicos se encuentran en un estado incipiente, pero con \\
& perspectivas muy prometedoras en diseño y manufactura aditiva de \\
componentes personalizados, así como sistemas auto-lubricantes que & prometen mejores resultados en comparación con sistemas actualmente \\
& ofertados en el mercado. El desarrollo de materiales como polímeros de \\
ultra alto peso molecular modificados con nanotubos de carbón, así \\
como superficies funcionalizadas con recubrimientos a base de carbón \\
y compuestos tipo diamante, que presentan características auto- \\
lubricantes, podrían incrementar la durabilidad de los implantes, \\
mejorando la calidad de vida para los pacientes.
\end{tabular}




\section{PRESENT \& FUTURE CHALLENGES IN KNEE AND HIP IMPLANTS}

\begin{tabular}{ll}
\hline KEYWORDS & ABSTRACT \\
\hline Knee arthroplasty & In this work, a literature analysis complemented by interviews of \\
Hip arthroplasty, & stakeholders and physicians specialized in knee and hip arthroplasty \\
Total joint replacements & was carried out to identify the most recent advances in these topics \\
3D printing & and the challenges and future perspectives that arise both in the \\
Additive manufacturing & surgical field and in the development of total joint replacements and \\
Surface functionalization & their materials. Mexico has plenty of clinical experience in prostheses \\
Self-lubricating. & implantation for knee, hip, shoulder, hand, and ankle joints. Likewise, \\
& the clinical experience in the placement of maxilo- and craniofacial \\
implants is extensive. However, the National Symposium - & Orthopedic Prosthesis: Current Status in Mexico \\
& https://protesismexico.com/) showed an imperative need to \\
& customize and design implants adapted to the Mexican population. In \\
& Mexico, the research of materials for orthopedic implants is in an \\
incipient stage, but with up-and-coming prospects in design and & additive manufacturing of customized components and self- \\
lubricating systems promise better results than currently offered \\
devices. The development of ultra-high molecular weight \\
polyethylene modified with carbon nanostructures and functionalized \\
surfaces with carbon-based coatings and diamond-like compounds \\
that present self-lubricating characteristics could increase the \\
durability of implants, improving the quality of life for many patients.
\end{tabular}

\section{INTRODUCCIÓN}

La artroplastia total de rodilla y cadera (ATR y ATC, respectivamente) se consideran las cirugías más exitosas en ortopedia debido a sus resultados notables, duraderos y reproducibles, que permiten devolverle movilidad e independencia a los pacientes intervenidos [1]. Estos procedimientos reconstructivos no solo alivian el dolor, sino que también mejoran la calidad de vida de las personas afectadas por artritis severa, artritis reumatoide (AR) y osteoartritis (OA). Según la Organización Mundial de la Salud, más de 23 millones de personas viven con AR, y los expertos predicen que este número se duplicará en 2030 [2]. De acuerdo a la Asociación Mexicana de Familiares y Pacientes con Artritis Reumatoide (AMEPAR), la AR es una enfermedad que predomina en el sexo femenino con un promedio de edad a partir de los 45 años, pero también puede aparecer en pacientes jóvenes.
En México existen datos registrados de pacientes con inicio de enfermedad a los 12 años de edad [3, 4]. Las enfermedades reumáticas afectan a aproximadamente 10 millones de personas en México, siendo la AR y la OA las dos más comunes, pues afectan a 1 y 8 millones de personas respectivamente. La población más afectada por padecimientos artríticos pertenece a niveles socioeconómicos medios y bajos, lo cual implica altos costos para la Secretaría de Salud, cuyo compromiso es garantizar el acceso a un tratamiento adecuado.

La OA es una enfermedad crónica caracterizada por mucho dolor e inhabilidad física, afectando principalmente a la rodilla, seguido por la cadera, sobre todo en la población adulta. Este padecimiento con mayor prevalencia y morbilidad en la población adulta de la tercera edad afecta alrededor del $60 \%$ de la población mexicana de este grupo; el cual presenta limitación física, movilidad reducida y utiliza con mayor 
frecuencia los servicios de salud a nivel mundial [5]. La prevalencia de OA de rodilla llega casi al $40 \%$ de la población mundial entre los 70 y 74 años de edad [5]. En Canadá se estima que para el 2026, más de 6 millones de canadienses padecerán artritis mientras que en los Estados Unidos de América (EE. UU.) y se prevé que en el año 2030 existan cerca de 67 millones de personas afectadas por este padecimiento. Los padecimientos artríticos (artrosis, artritis reumatoide $y$ artritis postraumática) han acompañado al ser humano a lo largo de la historia, siendo tratados básicamente con medicamentos y medidas generales paliativas [3]. Sin embargo, desde hace 70 años, en el caso de la cadera, y desde hace 60 años en el caso de la rodilla, se iniciaron los diseños modernos de reemplazos articulares para sustituir estas articulaciones [3].

El uso de estas prótesis a nivel mundial aumenta año con año, reflejando las cifras descritas para la prevalencia e incidencia de estos padecimientos. En el año 2003 se efectuaron 402,100 artroplastias totales primarias de rodilla sólo en los EE. UU.; en 2008, el número ascendía a más de 630 mil reemplazos de rodilla implantados [6], mientras que en 2017 se colocaron 1.6 millones de implantes de rodilla y cadera en EE. UU. (figura 1) [7] y se estima que para el año 2030 el incremento será del $673 \%$ [3].

En México existen pocas estadísticas, pero los datos disponibles en la Dirección General de Información en Salud (DGIS) indican que el número de reemplazos articulares de rodilla es importante (figura 2). Estos números, sin embargo, no reflejan de manera precisa las necesidades reales del país que pueden ser muy superiores, si no el número de intervenciones financiadas por el estado mexicano para ese año específico.

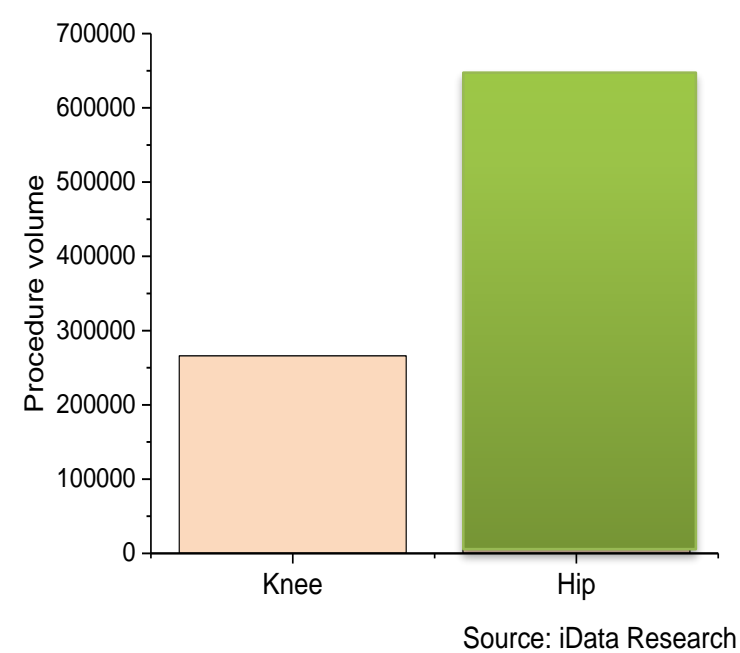

Figura 1. Artroplastias de rodilla y cadera realizadas en EE. UU. en 2017 [7].

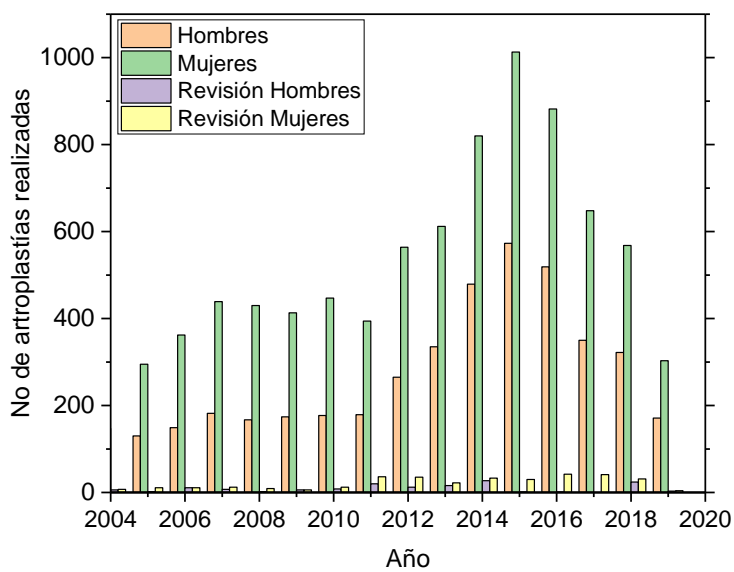

Figura 2. Número de artroplastias de rodilla primarias realizadas en México en hospitales públicos (DGIS) [8].

De acuerdo a diversos estudios, el $95 \%$ de estos implantes tiene una durabilidad de aproximada de 10 años. Asimismo, varios registros estadounidenses han confirmado ahora que la mayoría de las prótesis de rodilla y cadera que se utilizan actualmente pueden sobrevivir durante más de dos décadas [1]. Esto depende en gran medida de las condiciones y la actividad física del paciente. Muchos factores pueden influir en el éxito de la cirugía de reemplazo articular, incluida la selección del paciente, el diseño de la prótesis, la extensión del daño en la articulación, la precisión de la técnica 
quirúrgica con respecto al equilibrio de los tejidos blandos y la alineación de las extremidades, además de la eficacia del programa de rehabilitación posoperatoria [1]. En México, la mayoría de los implantes utilizados por el sector salud son importados. Aunado al precio elevado de las prótesis y la dependencia de tecnología extranjera, hay una limitación de los tamaños adecuados para la anatomía mexicana. Debido a que los implantes fueron diseñados para una población con características distintas a la población mexicana, sólo algunos de los tamaños de las prótesis se adaptan a la anatomía del paciente mexicano y en ocasiones hay que combinar las tallas de los componentes para un acople correcto, lo cual no es permitido por todas las compañías comercializadoras de implantes; los tamaños correctos de los componentes promueven una cinemática adecuada y pueden disminuir el dolor y la necesidad de revisión [9].

El objetivo del presente análisis documental es identificar los aspectos y avances más relevantes e integrarlos en el desarrollo de implantes ortopédicos (p.ej., rodilla y cadera) para su uso y aplicación en México. Este análisis documental condensa algunos datos y la opinión clínica de médicos especialistas participantes en el 1er Simposio nacional Prótesis Ortopédicas en México: Estatus Actual, el cual se llevó a cabo de manera virtual del 21 al 24 de septiembre de 2020.

\section{PRÓTESIS TOTAL DE RODILLA}

La artroplastia total de rodilla (ATR) es una opción quirúrgica que reemplaza la articulación de la rodilla con componentes artificiales (implantes metálicos y plásticos) y que ha demostrado una alta efectividad mejorando las condiciones físicas del paciente. En la artroplastia de rodilla se reemplaza parcial o totalmente esta articulación; es un procedimiento donde se extrae el tejido y hueso dañado de la parte baja del fémur, la parte superior de la tibia, y la patela o rótula; estas zonas son reemplazadas por un implante artificial que se integra a la rodilla. En general el procedimiento de colocación de la prótesis total de rodilla toma en algunos casos $\sim 2 \mathrm{~h}$. El procedimiento incluye la aplicación de la anestesia, la incisión, el corte en los huesos de la zona dañada, aplicación del cemento, la colocación de los diferentes componentes, y el cierre de la incisión. La ATR es necesaria cuando la articulación de la rodilla está desgastada o dañada a tal grado que la movilidad es reducida y la persona experimenta dolor, incluso cuando descansa $[3,5,10]$.

El diagnóstico más común para requerir una ATR es la artritis, específicamente la osteoartritis. Otras afecciones que causan daño severo a la rodilla también incluyen la artritis reumatoide, la hemofilia, la gota, trastornos que causan un crecimiento óseo inusual (displasias óseas), muerte del hueso en la articulación de la rodilla después de problemas de suministro de sangre (necrosis avascular), traumatismo, cáncer, deformidad de la rodilla con dolor y pérdida del cartílago $[5,11]$.

El número de ATR realizados a nivel mundial ha experimentado un crecimiento continuo a lo largo de los años; en 2011, se realizaron 1.324.000 procedimientos de ATR primarios y de revisión en sólo 18 países [11]. El número de ATR en los EE.UU. se ha duplicado en los últimos diez años $[12,13]$. De manera conservadora, el número de ATR primarias probablemente aumentará en un $143 \%$ para 2050 , alcanzando alrededor de 1,5 millones de casos/año solo en los EE. UU. [14, 15]. Otros países, como Austria, han experimentado un crecimiento continuo de alrededor del $13 \%$ anual entre 2009 y 2015 [16]. En México no hay un registro nacional 
para los pacientes que reciben una prótesis de rodilla. Sin embargo, se estima que las instituciones de salud pública como el Instituto de Seguridad y Servicios Sociales para Trabajadores del Estado (ISSSTE) adquieren anualmente un promedio de 700 prótesis de rodilla, todas de fabricación extranjera.

El primer implante de rodilla, específicamente de los cóndilos femorales, fue realizado en los años 40 por Smith y Petersen quienes diseñaron un modelo metálico para cubrir los cóndilos femorales, mientras que en los años 50 se realizó el primer intento para la sustitución de plataformas tibiales por McKeever y Macintosh [4, 17]. Sin embargo, debido a la complejidad de la articulación y sus movimientos, ambos intentos probaron ser un fracaso. La historia de las prótesis modernas y exitosas comienza en 1971, cuando Gunston introdujo una prótesis de baja fricción con dos superficies de acero que articulaban contra una superficie de polietileno de alta densidad [4]. En 1973 fue colocada la primera prótesis total condilar diseñada por Install y llamada "rodilla estabilizadora total condilar posterior", diseño en el cual se basan muchas de las prótesis actuales [17].

Los reemplazos totales de rodilla son dispositivos ortopédicos que están diseñados para ser operativos durante toda la vida del paciente a partir de la colocación. Sin embargo, un número considerable de implantes experimenta una falla prematura y necesita ser reemplazado (cirugías de revisión). La vida útil estimada para una rodilla artificial es de 15 a 20 años, aunque algunos implantes han mostrado vidas útiles más prolongadas, así como más reducidas. Las estadísticas indican que el $90 \%$ de los implantes de rodilla sobreviven los primeros 10 años, mientras que el $80 \%$ de los implantes sobreviven después de los 20 años, el porcentaje de supervivencia del implante disminuye significativamente después de ese tiempo [18]. Una prótesis total de rodilla está compuesta por tres partes básicas: 1) el componente tibial del reemplazo de rodilla se encuentra en la parte superior del hueso de la espinilla; 2) el componente femoral que encaja en el extremo del hueso del muslo; 3) y entre estos implantes metálicos hay un componente polimérico llamado espaciador (figura 3) [19]. Asimismo, existe un cuarto componente que se inserta en la patela. Los implantes de rodilla típicos, seleccionados por el médico cirujano de un rango limitado de tamaños, requieren cierta adaptación de los huesos del paciente a los contornos del implante.

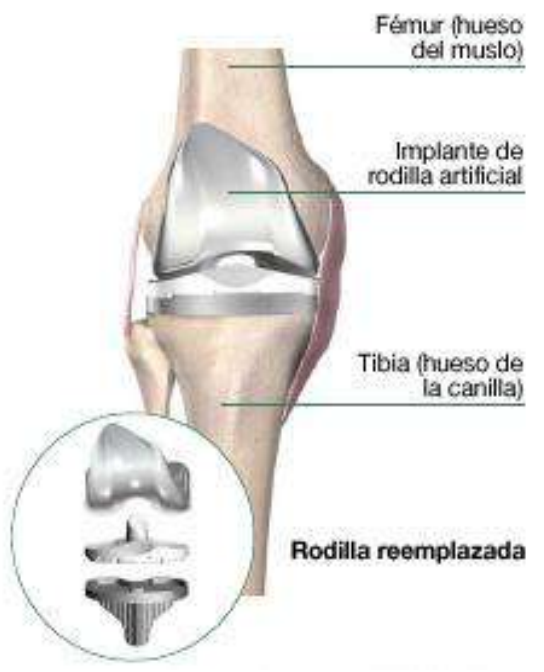

Figura 3. Componentes de la prótesis total de rodilla con diseño estándar [20][21]

Hoy en día, el número de modelos existentes en el mercado es muy elevado. Entre los principales fabricantes de estas prótesis se encuentran: Zimmer Biomet, Depuy Synthes Orthopedics, Smith \& Nephew, Inc., Stryker Orthopaedics y Wright Medical Group. La prótesis es seleccionada por el cirujano de acuerdo a las características propias del paciente, como lo son la edad, el peso, el nivel de actividad física, el estado de los ligamentos y la calidad del hueso [22]. Las prótesis de rodilla estándar están clasificadas de acuerdo 
a la superficie articular que reemplazan (unicompartimentales, bicompartimentales o patelofemoral y tricompartimentales $\mathrm{o}$ prótesis total de rodilla), el sistema de fijación empleado, y el grado de restricción mecánica que ofrecen (prótesis no restringida, prótesis semi-restringida y completamente restringidas) [6]. La figura 4 muestra las variaciones de diseño del Modelo Journey II de la marca Smith \& Nephew en función del grado de restricción mecánica de la prótesis [23]. El grado de restricción mecánica afecta el diseño del componente femoral, del componente tibial y del espaciador de UHMWPE.

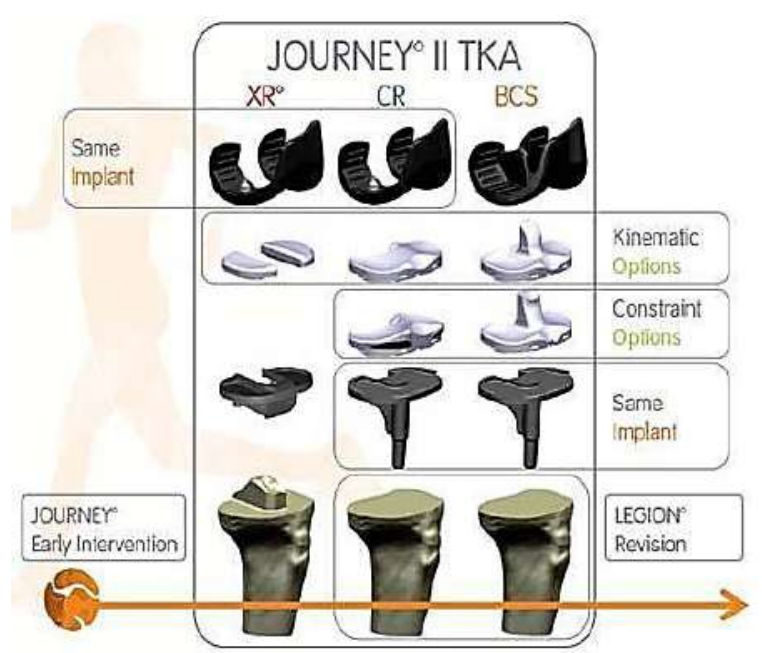

Figura 4. Modelo Journey II TKA de Smith and Nephew. Bi-Cruciate Retaining Knee System [23]

Preguntas cruciales en este tipo de cirugías pueden plantearse, por ejemplo, ¿cuáles son los aspectos principales a considerar para garantizar la integridad de los componentes del implante y el bienestar del paciente?, y ¿qué se puede hacer para mejorar el procedimiento y el resultado de la cirugía? Esto se responde en parte con los avances recientes en el área clínica que se orientan al desarrollo de procesos quirúrgicos más precisos, p.ej., cirugías asistidas con sistemas y brazos robóticos [24], así como a la personalización de implantes que permiten diseñar componentes específicos a la anatomía del paciente $[25,26]$. Esto ha sido realizado y reportado utilizando el procesamiento digital de imágenes de tomografía computarizada $[27,28]$ con la finalidad de asistir la planeación de la intervención quirúrgica, lo que ha impactado en la preservación del hueso femoral a intervenir y al mejoramiento en la integración del hueso cortical de la tibia (o preservar hueso sano). La personalización incluye también el diseño y desarrollo de las herramientas y material quirúrgico a la medida, lo cual permite restaurar más adecuadamente la anatomía del paciente, la posición central de la articulación y el funcionamiento normal de la articulación.

Otro enfoque está basado en garantizar la adaptabilidad del implante en la articulación del paciente; esto está muy ligado con la resistencia y durabilidad del implante, ya que una mala alineación puede causar desgaste prematuro en las superficies de cada uno de sus componentes y el daño del implante. Es crucial mejorar el balance de tejido, debido a que esto garantiza el acople y el buen desempeño del implante a largo plazo, ya que $\sim 25 \%$ de las revisiones de rodilla son debidas a inestabilidad, mala rotación y mala alineación según lo reportado por Martin Roche et al. [29] y sus referencias [30, 31]. Otros factores que conllevan a una revisión de implantes de rodilla son infecciones, el aflojamiento aséptico y fracturas [32]. La alineación del implante se realiza eficazmente a través de su colocación asegurando la alineación y balanceo de esfuerzos en los ligamentos medial y lateral, en los últimos adelantos, esto se realiza utilizando sensores de fuerza que monitorean de manera dinámica este procedimiento [33, 34]. 


\section{PRÓTESIS TOTAL DE CADERA}

La cadera es una de las articulaciones más grandes del cuerpo. Es una articulación de tipo rótula. La cavidad está formada por el acetábulo, que forma parte del hueso de la pelvis. La cabeza femoral es el extremo superior del fémur, y tanto el acetábulo como la cabeza femoral están cubiertas con cartílago articular, que amortigua los extremos de los huesos y les permite moverse con facilidad. Un tejido delgado llamado membrana sinovial rodea la articulación de la cadera; en una cadera sana, esta membrana produce una pequeña cantidad de líquido que lubrica el cartílago y elimina casi toda la fricción durante el movimiento de la cadera.

La causa más común de discapacidad y dolor crónico de cadera es la artritis. La cirugía de reemplazo de cadera es una de las operaciones más exitosas de toda la medicina [35]. Los primeros intentos registrados de reemplazo de cadera ocurrieron en Alemania en 1891, donde se utilizó el marfil para reemplazar las cabezas femorales de pacientes cuyas articulaciones de cadera habían sido destruidas por tuberculosis [36]. Posteriormente, los cirujanos experimentaron con la artroplastia interposicional a finales del siglo XIX y principios del XX, que consistía en colocar varios tejidos (fascia lata, piel, submucosa de la vejiga de cerdo) entre las superficies articulares de la cadera artrítica [36]. En 1925, el cirujano estadounidense Marius Smith-Petersen creó la primera artroplastia con molde fabricada en vidrio (Pyrex). A pesar de que el vidrio es un material biocompatible, no resistió los esfuerzos de la articulación de la cadera y se fracturó; basado en este diseño Marius SmithPetersen trabajó con Philip Wiles para desarrollar el primer reemplazo total de cadera hecho de acero inoxidable que se ajustó al hueso con pernos y tornillos [36].
Desde principios de la década de 1960, las mejoras en las técnicas y la tecnología quirúrgica de reemplazo de articulaciones han aumentado en gran medida la eficacia del reemplazo total de cadera. Sir John Charnly, cirujano ortopédico considerado el padre de la ATC moderna, diseñó a principios de la década de los 60s un reemplazo articular de cadera que en principio, es idéntica a las prótesis que se utilizan en la actualidad [36]. Las prótesis de cadera constan de cuatro componentes: un vástago femoral de metal, una cabeza femoral cerámica o metálica, un componente acetabular metálico y un espaciador plástico, cerámico o metálico que se inserta entre la cabeza femoral y el componente acetabular para permitir un movimiento suave (Figura 5).
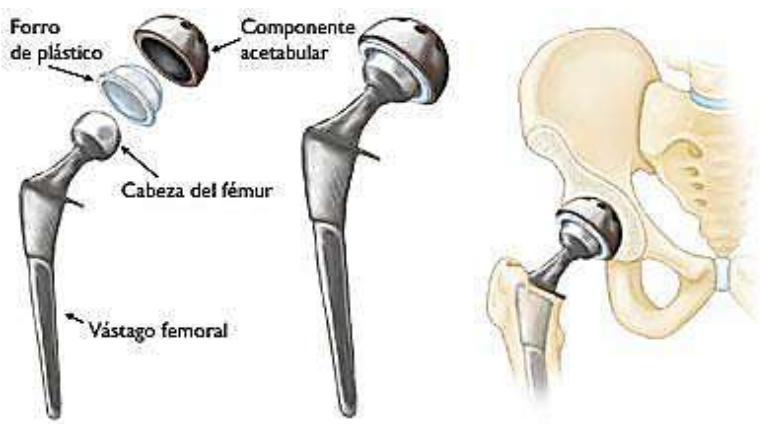

Figura 5. Componentes individuales del reemplazo total de cadera (izquierda), componentes unidos (centro), prótesis colocada (derecha) [37]

Según la Agencia para la Investigación y la Calidad de la Atención Médica, cada año se realizan más de 450,000 reemplazos totales de cadera en los EE. UU. [38]. En México, los datos no son tan elevados; de acuerdo a la DGIS, en 2016 se registraron sólo 2445 procedimientos para reemplazos totales de cadera. Sin embargo, se desconoce si dicho número obedece a una disponibilidad presupuestal, disponibilidad de recursos hospitalarios o a un padecimiento real. 


\section{MATERIALES UTILIZADOS EN LA FABRICACIÓN DE PRÓTESIS}

Los materiales utilizados en la fabricación de las prótesis han sido los mismos durante más de 20 años (Tabla 1) [39]. A pesar de que en ambos reemplazos articulares los materiales han variado poco en esencia, los materiales seleccionados para cada componente han ido modificándose con el tiempo basado en los resultados clínicos obtenidos.

Tabla 1. Biomateriales utilizados en prótesis de rodilla y cadera (adaptado de [14] y [37])

\begin{tabular}{|l|l|}
\hline \multicolumn{1}{|c|}{ MATERIAL } & \multicolumn{1}{c|}{ APLICACIÓN } \\
\hline $\begin{array}{l}\text { Aleaciones de cobalto } \\
\text { Co-Cr-Mo (fundido) } \\
\text { Co-Ni-Cr-Mo (forjado) }\end{array}$ & $\begin{array}{l}\text { Recubrimientos porosos y componentes femorales y } \\
\text { tibiales de la prótesis de rodilla. } \\
\text { Componente femoral y acetabular de la prótesis de } \\
\text { cadera. }\end{array}$ \\
\hline Ti6Al4V (Ti64) & $\begin{array}{l}\text { Componentes femorales de las prótesis de rodilla y cadera } \\
\text { y componentes tibiales, recubrimientos porosos. }\end{array}$ \\
\hline $\begin{array}{l}\text { Polietileno de ultra alto peso molecular } \\
\text { (ingl. UHMWPE) }\end{array}$ & $\begin{array}{l}\text { Espaciadores para prótesis de rodilla y cadera } \\
\text { y componente patelar. }\end{array}$ \\
\hline Alúmina & Cabeza femoral y espaciador de prótesis de cadera \\
\hline Alúmina/Zirconia & $\begin{array}{l}\text { Cabeza femoral de la prótesis de cadera y espaciador de la } \\
\text { prótesis de cadera }\end{array}$ \\
\hline Hidroxiapatita & Recubrimiento de vástagos femorales \\
\hline
\end{tabular}

\subsection{Materiales metálicos}

Hoy en día, las aleaciones base cobalto y base titanio son las principales aleaciones utilizadas en la fabricación de los componentes metálicos de ambas prótesis articulares. Por ejemplo, los componentes femorales de las prótesis de rodilla son generalmente fabricadas de la aleación cromo-cobalto conocida como Vitallium $\AA$ que contiene $30 \%$ de cromo, $7 \%$ molibdeno, cobalto y níquel, entre otros. Este material es resistente al rayado y tiene una excelente resistencia al desgaste, posee una alta resistencia a la corrosión y formación de picaduras y hendiduras, y se considera, en general, seguro para su uso en reemplazos de rodilla [13].

La aleación Ti6Al4V (ELI o grado 23 de acuerdo a la clasificación de aleaciones de titanio) (Ti64) es también utilizada para la fabricación de implantes femorales de rodilla. Esta aleación es la versión de mayor pureza de Ti64 y tiene una excelente combinación entre resistencia mecánica, ligereza, resistencia a la corrosión y tenacidad. Sin embargo, es menos resistente al desgaste que las cromo-cobalto y se raya fácilmente; por tal motivo, su uso debe ser valorado para cada 
paciente para el componente femoral. En cambio, su uso es ideal para los platos tibiales de la prótesis de rodilla, ya que esta aleación es más ligera y su módulo elástico es más cercano al del hueso que el Vitallium®.

Por otro lado, en las prótesis totales de cadera hay más variación en cuanto a los materiales. De los cuatro componentes, el vástago y el componente acetabular son fabricados con aleaciones base cobalto $\mathrm{y}$ base titanio, mientras que la cabeza femoral y el espaciador pueden ser fabricados de aleaciones base cobalto $\mathrm{u}$ otros materiales que se describirán más adelante.

Los metales tradicionales para las prótesis totales de cadera son los aceros inoxidables, las aleaciones de titanio (p.ej., Ti64) y, principalmente, las aleaciones de cobaltocromo-molibdeno. Esta última tiene una buena resistencia a la corrosión en comparación con otros metales, alta tenacidad, alta resistencia al desgaste y mayor dureza $(\mathrm{HV}=350)$ que otros metales $\mathrm{y}$ polímeros [40]. La articulación de metal sobre metal (MoM metal-on metal) donde tanto la cabeza femoral como el espaciador son metálicos, se produce típicamente a partir de aleaciones de cobalto-cromo-molibdeno (CoCrMo), las cuales se componen de 58,9 a $69,5 \%$ de Co, 27,0 a $30 \%$ de $\mathrm{Cr}, 5,0$ a 7,0 \% de Mo y una pequeña cantidad de otros elementos (Mn, Si, Ni, Fe y C) [40]. Ya que en el vástago no hay un riesgo elevado de desgaste, este componente puede fabricarse con Ti64.

\subsection{Materiales poliméricos}

Las superficies articulares o espaciadores de las prótesis de rodilla son fabricadas de polietileno de ultra alto peso molecular (ingl. Ultra High Molecular Weight Polyethylene UHMWPE), mientras que, en las prótesis de cadera, el espaciador puede ser fabricado de UHMWPE, CoCrMo, o materiales cerámicos. El UHMWPE es un polímero lineal semicristalino que se ha utilizado ampliamente en aplicaciones biomédicas en el campo ortopédico debido a su alta resistencia al desgaste, bajo coeficiente de fricción, alta resistencia al impacto, biocompatibilidad y alta tenacidad a la fractura [12, 41]. Charnley introdujo UHMWPE en 1962 para reemplazos totales de cadera [40]. Este polímero se crea mediante la polimerización de etileno. Cadenas excepcionalmente largas de polietileno forman UHMWPE, y cada molécula adicional mejora toda la estructura [42], lo que confiere propiedades físicas y mecánicas intrínsecas extraordinarias diseñadas para mejorar el rendimiento clínico del UHMWPE. Desde su introducción al campo ortopédico, el UHMWPE ha sido mejorado constantemente para disminuir su desgaste, el cual es inevitable debido al constante movimiento de los componentes metálicos sobre los espaciadores en ambas prótesis.

Existen varias generaciones de UHMWPE, cada una de ellas cuenta con mejoras que han sido comprobadas a nivel clínico. Sin embargo, estas mejoras en el material aún no son suficientes para las necesidades operativas de ambas prótesis. Entre los cambios más destacados se encuentran el polietileno reticulado, introducido en los años 70s [43]; el polietileno altamente reticulado, incluido en los años 2000s [44], y en los últimos años se ha visto la introducción de la vitamina $\mathrm{E}$ y diferentes tratamientos térmicos para disminuir la oxidación causada por la irradiación en el proceso de esterilización y mantener las propiedades mecánicas del UHMWPE en su estado virgen [44-47].

\subsection{Materiales cerámicos}

En las prótesis totales de cadera, hay dos componentes que pueden ser fabricados con materiales cerámicos: la cabeza femoral y el 
espaciador. Los materiales cerámicos se introdujeron en las prótesis totales de cadera desde hace más de veinte años con el fin de reemplazar al UHMWPE y evitar el desgaste de este. Aunque los cerámicos también poseen una excelente resistencia al desgaste, estos se fracturan con facilidad, lo que conlleva a una selección muy cautelosa de los cerámicos utilizados en esta industria [5]. Hasta el momento, solo hay tres cerámicos que se han utilizado en la fabricación de cabezas femorales y espaciadores, la alúmina, la zirconia y la alúmina reforzada con zirconia.

La alúmina se introdujo en los implantes THA en 1971 por Boutin, quien obtuvo buenos resultados clínicos al realizar un acoplamiento de cadera de alúmina sobre alúmina $[40,48]$. La alúmina es una de las principales cerámicas utilizadas en ATC, gracias a sus buenas propiedades tribológicas y dureza, lo que significa un bajo coeficiente de fricción y una alta resistencia al desgaste. Sin embargo, tiene una resistencia mecánica baja en tracción. El comportamiento de la alúmina está relacionado con diferentes aspectos, como la densidad, la pureza y el tamaño de grano [40]. El último, en particular, influye en la tasa de desgaste, ya que disminuye con el tamaño de grano más pequeño. Hoy en día, la alúmina conocida comercialmente como Biolox ${ }^{\circledR}$ forte (introducida en 1994 por Biomet Zimmer) es utilizada en reemplazos totales de cadera y presenta una densidad de $3.98 \mathrm{~g} / \mathrm{cm}^{3}$ y un tamaño de grano de $3.2 \mu \mathrm{m}$ [49].

Por otro lado, la zirconia tiene alta tenacidad, buenas propiedades mecánicas y una excelente resistencia al agrietamiento, principales razones que hicieron de la zirconia una alternativa para las prótesis totales de cadera. La primera generación de cabezas femorales de zirconia fue fabricada de zirconia parcialmente estabilizada con magnesia (MgPSZ), que no cumplía con los requisitos de resistencia al desgaste [40]. Por lo tanto, los desarrollos posteriores se centraron en el óxido estabilizador de itrio (Y-TZP), una cerámica que está completamente formada por granos de tamaño submicrónico (estándar actual para la aplicación clínica) [50]. Y-TZP se compone de granos tetragonales de tamaño inferior a $0,5 \mu \mathrm{m}$, cuya fracción retenida a temperatura ambiente depende del tamaño, la distribución y la concentración del óxido estabilizador de itrio [40, 51]. Dichos parámetros microestructurales definen las propiedades mecánicas del Y-TZP. Los granos tetragonales pueden transformarse en granos monoclínicos, produciendo una expansión de volumen del 3 al 4\%, que es la razón detrás de la tenacidad de la cerámica y su capacidad para disipar la energía de fractura [40]. Incluso si en la práctica clínica las cabezas femorales Y-TZP sólo se acoplaron con copas de UHMWPE, las pruebas realizadas con YTZP frente a alúmina arrojaron resultados positivos; sin embargo, la Y-TZP tiende a envejecer en presencia de agua, por lo que su uso a nivel clínico sigue siendo cuestionable [42].

Finalmente, en la década de 2000, la primera alúmina endurecida con zirconia (ZTA por sus siglas en inglés) fue introducida clínicamente con el nombre comercial de Biolox ${ }^{\circledR}$ Delta; este material proporciona una elevada resistencia al inicio del agrietamiento y a la propagación del agrietamiento [42]. ZTA combina las mejores características tanto de la alúmina como de la zirconia: la resistencia y tenacidad de la alúmina y la excelente resistencia al desgaste, estabilidad química e hidrotermal de la alúmina. Sin embargo, los compuestos todavía exhiben un envejecimiento significativo atribuible a la presencia de alúmina [52]. 


\section{RETOS ACTUALES EN IMPLANTES ORTOPÉDICOS}

\subsection{Retos en la artroplastia de rodilla}

\subsubsection{Prótesis de rodilla para adulto - el estatus en México}

En México, la Guía de Práctica Clínica para el Tratamiento con Artroplastia Total de Rodilla está diseñada para dar atención a pacientes de 60 años de edad con osteoartritis y daño estructural severo [3]. El paciente es candidato a reemplazo articular si presenta dolor sin mejoría con algún tratamiento, además de presentar características radiográficas grado 3 y 4 en la escala de Kellgren-Lawrence [53]. Algunas contraindicaciones para el reemplazo de rodilla son, p.ej., condiciones médicas que representen un procedimiento anestésico no seguro, malas condiciones de la piel, historial de procesos infecciosos, enfermedad vascular severa, artrodesis de rodilla funcional, artropatía neuropática, obesidad mórbida y/o disfunción mental [5]. De acuerdo a datos clínicos presentados en el Simposio - Prótesis ortopédicas, los mejores resultados se obtienen en pacientes mayores de 60 años con deformidad angular menor a $15^{\circ}$ de valgo o $10^{\circ}$ en varo con un IMC menor a 30 . Sin embargo, para esto se requiere de una medición y alineación precisa de los componentes, así como de la fijación segura con cemento (polimetilmetacrilato), además de un balance de los tejidos blandos y del funcionamiento rotuliano. Otro reto importante durante el procedimiento quirúrgico es la prevención de la necrosis térmica que puede provocar la polimerización del polimetilmetacrilato, proceso en el cual la temperatura puede alcanzar hasta los $130{ }^{\circ} \mathrm{C}$. Por otra parte, las prótesis que se colocan en pacientes mexicanos son prótesis que fueron diseñadas de manera estándar (chico, mediano, o grande) para la anatomía del cuerpo de un estadounidense o un europeo, lo que resulta en una adaptación del implante y la remoción de hueso sano y dificultades para la rehabilitación del paciente.

\subsubsection{Prótesis de rodilla para niño - El estatus en México}

Los implantes para jóvenes y niños todavía se encuentran en un estado incipiente y el reto reside en que sus huesos siguen en crecimiento, lo que dificulta la estrategia para el óptimo funcionamiento en pacientes que siguen en desarrollo. Los implantes más comunes son componentes femoral y tibial con un solo grado de libertad. El componente tibial se compone de una pieza que tiene un tornillo ajustable con una rosca, ver Figura 6. Este mecanismo se ha diseñado para poder incrementar la longitud de la prótesis a medida que el paciente crece. Lo que implica que el paciente deba ser intervenido quirúrgicamente cada vez que se requiera incrementar la longitud de la prótesis causando dolor; esto sin considerar que se pueden presentar complicaciones por infecciones. De acuerdo a la opinión clínica de los participantes del Simposio Nacional Prótesis ortopédicas: Estatus actual en México https://protesismexico.com/, hay una clara necesidad de personalización de implantes y dispositivos para niños, quienes presentan diversas lesiones y problemas causados por osteosarcoma. En el mercado sólo existen prótesis de este tipo con tamaños estándar que no han sido diseñadas para la anatomía del mexicano. Según datos de la Secretaría de Salud, en México se registran alrededor de 100 a 150 casos de osteosarcoma por año, siendo el tipo de cáncer más frecuente en niños y adolescentes [54]. 


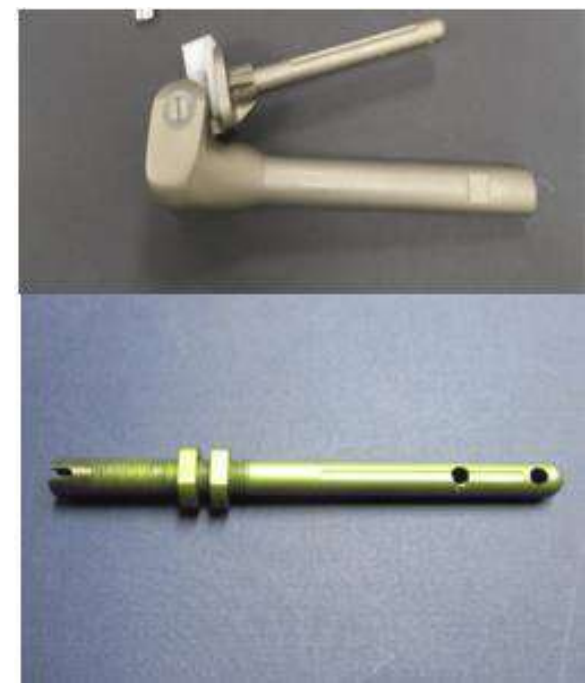

Figura 6. Implante de Ti64 para reemplazo de rodilla para niño (superior) componente tibial y femoral y (inferior) vástago del componente tibial para incrementar el tamaño del implante.

\subsection{Retos en la artroplastia de cadera}

La artroplastia de cadera es un procedimiento con muy buenos resultados clínicos. Actualmente, los espaciadores de polietileno altamente reticulado y los espaciadores cerámicos han proporcionado excelentes resultados a mediano y largo plazo [55]. Por lo tanto, las nuevas investigaciones se centran en mejoras funcionales de los resultados posoperatorios. Los cirujanos de cadera deben conocer los avances en las técnicas quirúrgicas y la evidencia detrás de la selección de implantes y el material de los espaciadores, así como la mejora continua en los resultados funcionales de este procedimiento para maximizar la longevidad de las prótesis implantadas [56]. Deben tenerse en cuenta hallazgos como la mejora en el rango de movimiento (ingl. Range of Motion - ROM), resultante del uso de cabezas de $36 \mathrm{~mm}$ en comparación con cabezas de 28 $\mathrm{mm}$, así como las diferencias en el desempeño de las prótesis derivadas de las diferencias entre las actividades cotidianas de pacientes con diferentes diagnósticos [57]. Por ejemplo, en la región asiática, las personas pasan mucho más tiempo con las piernas cruzadas, en cuclillas y arrodilladas, lo que conlleva a otro tipo de fallas en los implantes de cadera. Cuando los pacientes realizan actividades que aumentan el ROM después de una ATC, puede producirse un choque entre el espaciador y el vástago femoral. En el estudio realizado por W.Y. Shon et al. [58], se demostró que la aparición de pinzamiento (Figura 7) aumenta significativamente si el diagnóstico de revisión es dislocación, si el componente tiene un diámetro exterior grande o un borde elevado y si el componente femoral tiene un cuello con borde extendido. La alta incidencia de pinzamiento después de una ATC implica que los resultados de los diseños actuales de reemplazos totales de cadera carecen de la capacidad para realizar todas las actividades físicas diarias [58]. Los cambios en el diseño del implante (p.ej., evitar cabezas con faldón, maximizar la relación cabeza-cuello, usar un revestimiento acetabular biselado y una sección transversal del cuello trapezoidal) pueden ayudar a reducir la prevalencia de pinzamiento $[56,58$, 59].

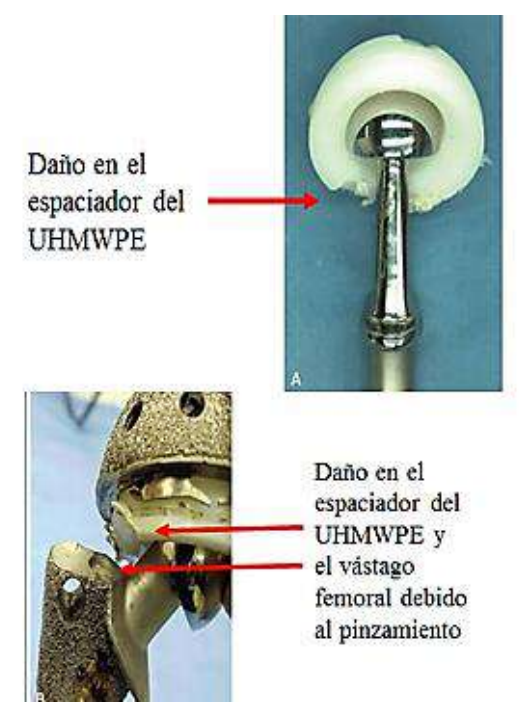

Figura 7. Componentes acetabulares y femorales recuperados de un paciente masculino de 43 años que revela daño en el espaciador de UHMWPE por impacto entre el borde del espaciador y el cuello del vástago femoral. Adaptada de [58, 59] 
En México, los mayores retos a nivel clínico son la implementación de métodos asistidos con robots (de costo accesible) para realizar procedimientos quirúrgicos mínimos invasivos. Por otra parte, existen retos a nivel global en el desarrollo de componentes y estructuras mecánicamente más afines al hueso cortical y trabecular, así como de materiales biocompatibles (polimetilmetacrilato, polietileno de ultraalto peso molecular) más resistentes al desgaste para su aplicación en las prótesis de cadera.

\subsection{Avances y retos actuales en el desarrollo de materiales para implantes ortopédicos}

\subsubsection{Cementos óseos}

En el caso de prótesis para reemplazo total de cadera, el polimetilmetacrilato (PMMA) utilizado para fijar el implante (cementado) ha dado muy buenos resultados, sin embargo existe evidencia que relaciona la formación de partículas (Debris) con el daño y resorción ósea local o general (osteólisis) [60]. Debido a esto se han desarrollado prótesis nocementadas que han presentado otros problemas como atrofia cortical, dolor o la osteólisis asociada al polietileno de ultra alto peso molecular utilizado en el implante. En México, datos recientes indican que existen pacientes que tienen ambos sistemas de fijación para prótesis de cadera (cementadas y no cementadas) [61]. Por su parte, la funcionalización de cementos óseos sigue siendo un tema de investigación con diversos enfoques, por ejemplo con la hidroxiapatita (HA), sin embargo algunos estudios muestran que cementos no cumplen la resistencia mecánica mínima con la adición de este material bioactivo [62]. La disminución de las propiedades mecánicas del compuesto están relacionadas con la aglomeración de partículas de HA. Otras investigaciones recientes muestran que compuestos de
PMMA con un contenido de microesferas de HA de hasta $40 \%$ pueden conservar su resistencia a la compresión y mejoran la osteoconductividad [63]. La adición de HA para mejorar p.ej. la radio-opacidad de los cementos óseos sigue siendo un tema de interés.

\subsubsection{Materiales metálicos}

Dos grandes retos han dictado la investigación en el ámbito de materiales metálicos: la correcta transferencia de esfuerzos entre los componentes metálicos y el hueso, y la toxicidad de iones y partículas de desgaste metálicos.

Los materiales metálicos tienen un alto módulo de elasticidad (CoCrMo tiene un módulo de Young de $240 \mathrm{GPa}$ mientras que el Ti64 tiene un módulo de $110 \mathrm{GPa}$ ), lo que limita la distribución de la tensión del implante al hueso (hueso trabecular $3 \mathrm{GPa}$ y hueso cortical 12-17 GPa) [64]. Por tanto, se están desarrollando nuevos componentes metálicos con menor módulo elástico y mayor resistencia a la corrosión y al desgaste [38]. Un ejemplo es el desarrollo de las aleaciones base Ti de tipo $\beta$ con elementos no citotóxicos y con módulos de elasticidad (Young) inferiores (alrededor de los $40 \mathrm{GPa}$ ).

Entre las aleaciones Ti- $\beta$ más estudiadas se encuentran los sistemas TiNb, TiTa, TiNbZr, TiNbSn [65]. Debido a la novedad de estas aleaciones, estas se han fabricado mediante técnicas de manufactura convencional como la forja y la fundición, y muy pocos esfuerzos para su síntesis o procesamiento se han llevado a cabo por medio de la Manufactura Aditiva (MA). Un inconveniente identificado en las aleaciones Ti- $\beta$ es su baja resistencia mecánica y la resistencia a la fatiga, lo que las hace poco apropiadas para aplicaciones de carga. Con el propósito de mejorar este comportamiento, estudios recientes se han centrado en desarrollar aleaciones base Ti con 
una matriz de fase $\beta$, reforzada con precipitados $\alpha$ y $\omega$, buscando conservar su módulo de Young [66]. Debido a la resorción ósea causada por la inadecuada transferencia de esfuerzos de la prótesis hacia el hueso, es crucial que el módulo de Young de estos sistemas de aleación permanezca en el rango de 10-30 GPa. Teniendo esto en cuenta, se podría diseñar una aleación que se adapte al comportamiento mecánico buscado de acuerdo a la aplicación del implante metálico $[38,67-70]$.

Hasta el momento, existen muy pocos estudios que aborden el diseño y evaluación de las aleaciones $\beta$-Ti en aplicaciones biomédicas [71]; en este sentido, es crucial definir los parámetros de procesamiento, las propiedades de composición, microestructurales y mecánicas que gobiernan la biocompatibilidad, osteointegración, corrosión y desgaste para garantizar el desempeño de los biomateriales metálicos.

Por otro lado, a pesar de que la aleación CoCrMo tiene una alta resistencia al desgaste, en los implantes de cadera MoM puede haber desprendimiento de partículas de desgaste de esta aleación, las cuales son transferidas al líquido sinovial donde son capaces de migrar a la sangre [40]. El cobalto y el cromo están presentes en el medio ambiente y en los alimentos y son necesarios para los seres humanos como oligoelementos en el cuerpo, pero son tóxicos en concentraciones elevadas [38]. Hay poco conocimiento sobre los efectos del Co y $\mathrm{Cr}$ circulantes en la sangre; los cuales pueden afectar principalmente funciones biológicas y celulares con efectos potenciales sobre el sistema inmunológico, mutagénesis y carcinogénesis [38]. Aunado a esto, las grandes diferencias entre el módulo elástico de esta aleación y el módulo elástico del hueso demuestran la necesidad de buscar materiales alternativos.

Otro ejemplo claro es el esfuerzo por reemplazar al Ti64, que a pesar de ser el biomaterial metálico más aceptado, ha generado algunos problemas debido a la liberación de iones Al y V, los cuales han sido asociados con el desarrollo de Alzheimer y neuropatías [38]. Además, la poca resistencia al desgaste de Ti64 conduce a la producción de partículas que se han vinculado con una mayor susceptibilidad a las convulsiones [71]. Sin embargo, dado que la vida útil de un implante metálico oscila entre los 10 a 20 años dependiendo de su manufactura, material, afectación del paciente y el uso de la misma, sus periodos de evaluación para determinar los efectos secundarios son también estudiados en periodos de tiempo similares.

Como alternativas para mejorar la biocompatibilidad del Ti64, algunas partes de los implantes actuales han sido protegidos con recubrimientos cerámicos; sin embargo, esto no resuelve completamente el problema al existir secciones del mismo que no pueden ser protegidas por su funcionalidad [72 -74]. Existe una continua investigación de nuevas aleaciones metálicas para su aplicación en prótesis para obtener una mejor biocompatibilidad junto con propiedades mecánicas superiores.

\subsubsection{Materiales poliméricos}

El UHMWPE sigue siendo la primera elección para superficies articulares de prótesis de rodilla gracias a sus buenas propiedades mecánicas y su alta resistencia al desgaste. Asimismo, este material es uno de los más comunes para la fabricación de espaciadores de prótesis totales de cadera (MoP - metal sobre plástico). Sin embargo, el aflojamiento aséptico como resultado de la osteólisis relacionada con los residuos de desgaste de UHMWPE es una de las causas de falla en los reemplazos totales de articulaciones (tanto en cadera como en rodilla) modernos [43, 75-78], lo que limita la longevidad del implante [12]. 
Algunas investigaciones recientes están dedicadas a la modificación del UHMWPE con materiales reforzantes, p.ej., nanotubos de carbono, nano-plaquetas de grafeno y óxidos de grafito y grafeno, que le confieren la capacidad auto-lubricante del material, mejora en su resistencia al desgaste [79-81], y reduce el envejecimiento (por oxidación) del material [82-91]. Recientemente se ha mostrado que superficies auto-lubricantes como recubrimientos tipo diamante (ingl. Diamond Like Carbon - DLC) o carburos de Ti, p.ej., c-TiC presentan un bajo coeficiente de fricción. El uso de pares tribológicos de polietileno reforzado con nanotubos de carbono (UHMWPE-MWCNTs) contra recubrimientos como c-TiC podría permitir disminuir el desgaste del polímero. En un estudio donde se analizó en un tribómetro tipo pin-on-disc el desgaste de UHMWPEMWCNT contra una superficie recubierta de c-TiC sin lubricantes, hubo una reducción hasta en un $43.7 \%$ en comparación con un par de referencia donde se no incluyen estos sistemas a pesar de que la rugosidad $(\mathrm{Ra}) \mathrm{del}$ recubrimiento era ligeramente más elevada que la sugerida para los implantes de rodilla en norma ASTM F2083 [92]. La Tabla 2 muestra la comparación de este sistema tribológico con el UHMWPE sin refuerzo cuando se desgasta contra una superficie de acero y una superficie recubierta con la superficie lubricante de c-TiC.

Tabla 2. Pérdida de peso y de volumen del sistema UHMWPE-MWCNTs vs. c-TiC después de 400,000 ciclos en pin-on-disk bajo movimiento reciprocante de $90^{\circ}$ con $1 \mathrm{~N}$ de carga normal y $2 \mathrm{~Hz}$ sin medio lubricante

\begin{tabular}{|c|c|c|c|c|}
\hline Par tribológico & $\begin{array}{c}\text { Peso } \\
\text { perdido } \\
(\mathbf{m g})\end{array}$ & $\begin{array}{c}\text { Volumen } \\
\text { perdido } \\
\left(\boldsymbol{\mu m}^{3}\right)\end{array}$ & Mejora en el desgaste & Volumen desgastado/Nm \\
\hline $\begin{array}{c}\text { UHMWPE vs } \\
\text { acero }\end{array}$ & 1.9 & 959,290 & Sistema de referencia & $\begin{array}{c}\text { UHMWPE vs. acero: } \\
6.056 \times 10^{-7} \mathrm{~mm}^{3} / \mathrm{Nm}\end{array}$ \\
\hline $\begin{array}{c}\text { UHMWPE- } \\
\text {-MWCNTs vs } \\
\text { acero }\end{array}$ & 1.2 & 623,274 & $\begin{array}{c}35.03 \% \text { - comparado con } \\
\text { UHMWPE-acero }\end{array}$ & $\begin{array}{c}\text { MWCNT-UHMWPE vs. } \\
\text { acero: } 3.935 \times 10^{-7} \mathrm{~mm}^{3} / \mathrm{Nm}\end{array}$ \\
\hline $\begin{array}{c}\text { UHMWPE- } \\
\text {-MWCNTs vs } \\
\text { c-TiC }\end{array}$ & 0.7 & 540,346 & $\begin{array}{c}43.7 \% \text { - comparado con } \\
\text { UHMWPE-acero } \\
13.31 \% \text { - comparado con } \\
\text { UHMWPE-MWCNTs-acero }\end{array}$ & $\begin{array}{c}\text { MWCNT-UHMWPE vs. c- } 3.4113 \times 10^{-7} \mathrm{~mm}^{3} / \mathrm{Nm} \\
\text { TiC }\end{array}$ \\
\hline
\end{tabular}

\subsubsection{La Manufactura Aditiva para el diseño de prótesis ortopédicas}

Otros desarrollos recientes de materiales están enfocados a la personalización de implantes para rodilla y cadera a través del diseño y de la implementación de nuevos procesos de manufactura, p.ej. la MA mejor conocida entre la comunidad como impresión en 3D [26, 93]. La MA, engloba un conjunto de tecnologías que permite la construcción de un objeto tridimensional de prácticamente cualquier forma a partir de una modelo digital (ver Tabla 3).

En particular, la MA se ha aplicado a varios sectores industriales incluyendo la manufactura de implantes ortopédicos. De hecho, en 2010 la agencia de administración de Alimentos y Medicamentos de los EE. UU. (ingl. Food and Drug Administration - FDA) aprobó el primer implante metálico producido 
por MA, y a la fecha se han aprobado más de 100 implantes distintos producidos por tecnologías aditivas que van desde craneales, cadera, rodilla y hasta columna [94]. Lo que deja claro que actualmente la MA es apta para la producción de dispositivos médicos, visto que la FDA en el año 2017 publicó una primera versión de directrices para el uso de la MA en la producción de dispositivos médicos [95].

Tabla 3. Clasificación de procesos y tecnologías de manufactura aditiva de acuerdo a la sociedad americana de pruebas y materiales (American Society for Testing and Materials ASTM) adaptado de [96]

\begin{tabular}{|c|c|c|c|}
\hline & Categoría & Tecnologías & Ventajas / Desventajas \\
\hline & $\begin{array}{l}\text { Fotopolimerización } \\
\text { Vat- } \\
\text { photopolymerization }\end{array}$ & $\begin{array}{ll}- & \text { SLA } \\
- & \text { DLP } \\
- & \text { CLIP }\end{array}$ & $\begin{array}{l}\text { - } \text { Alta velocidad de construcción } \\
\text { - } \text { Buena resolución } \\
\text { - } \text { Aurado con forma de láser } \\
\text { materiales }\end{array}$ \\
\hline & $\begin{array}{l}\text { Inyección de material } \\
\text { Material Jetting (MJ) }\end{array}$ & $\begin{array}{ll}\text { - } & \text { Polyjet } \\
\text { - } & \text { MJP }\end{array}$ & $\begin{array}{l}\text { - } \quad \text { Impresión multi-material } \\
\text { - } \quad \text { Buen acabado superficial } \\
\text { - } \text { Materiales de baja resistencia }\end{array}$ \\
\hline & $\begin{array}{l}\text { Extrusión de Material } \\
\text { Material Extrusion } \\
(M E)\end{array}$ & $\begin{array}{ll}- & \text { FDM } \\
- & \text { FFF } \\
- & \text { ADAM }\end{array}$ & $\begin{array}{ll}\text { - } & \text { Extrusor poco costoso } \\
\text { - } & \text { Impresión multi-material } \\
\text { - } & \text { Resolución limitada } \\
\text { - } & \text { Pobre acabado superficial }\end{array}$ \\
\hline & $\begin{array}{l}\text { Inyección de } \\
\text { aglutinante } \\
\text { Binder Jetting }(B J)\end{array}$ & $\begin{array}{ll}\text { - } & \mathrm{MJF} \\
\text { - } & \mathrm{SPJ}\end{array}$ & $\begin{array}{l}\text { - Impresión de objetos a todo color } \\
\text { - } \\
\text { - } \text { pequiere infiltración durante el } \\
\text { - } \quad \text { Grandia gama de materiales } \\
\end{array}$ \\
\hline & $\begin{array}{l}\text { Fusión de cama de } \\
\text { polvos } \\
\text { Powder Bed Fusion } \\
(P B F)\end{array}$ & $\begin{array}{ll}\text { - } & \text { SLS } \\
\text { - } & \text { SLM } \\
\text { - } & \text { DMLS } \\
\text { - } & \text { EBM }\end{array}$ & $\begin{array}{l}\text { - } \text { Gran exactitud y detalle } \\
\text { - } \text { Piezas completamente densas } \\
\text { - } \text { Alta resistencia } \\
\text { - } \text { Reciclado de polvo } \\
\text { Estructuras de soporte y anclaje }\end{array}$ \\
\hline & $\begin{array}{l}\text { Depósito por energía } \\
\text { directa } \\
\text { Direct Energy } \\
\text { Deposition (DED) }\end{array}$ & $\begin{array}{ll}- & \text { LENS } \\
- & \text { EBAM } \\
- & \text { LMD-w } \\
- & \text { WAAM }\end{array}$ & $\begin{array}{l}\text { - Alta tasa de depósito comparado } \\
\text { con procesos de cama de polvos } \\
\text { - } \quad \text { Reparación de partes } \\
\text { desgastadas/dañadas } \\
\text { - Impresión de materiales } \\
\text { - funcionales } \\
\text { - Requiere pos-procesamiento }\end{array}$ \\
\hline
\end{tabular}




\begin{tabular}{|l|l|l|l|}
\hline & & & \\
Laminación de hojas & $\bullet$ LOM & $\begin{array}{l}\text { Buen acabado superficial } \\
\text { Bajo costo de material, equipo y } \\
\text { Sheet Lamination }(S L)\end{array}$ & $\begin{array}{l}\text { pam } \\
\text { proceso }\end{array}$ \\
\hline
\end{tabular}

Ventajas:

\section{- Diseñar con alta complejidad:} libertad de diseño en partes con geometrías complejas y nuevos materiales, ideal para el diseño de implantes biomiméticos complejos, sin olvidar que se deben tomar en consideración los límites e implicaciones de cada tecnología (Tabla 3).

\section{- Acelerar el tiempo de} comercialización y los ciclos de innovación: ofrece una alternativa más rápida para pasar del concepto a la producción, además de reducir el tiempo y costo para el diseño, desarrollo y prueba de productos.

\section{- Reducción de pasos de producción:}

reduce la necesidad de grandes líneas de manufactura, ya que elimina la necesidad de algunos procesos, por lo tanto también disminuye el costo de manufactura.

- Mejora de desempeño por optimización: se pueden diseñar piezas con nuevas propiedades, características o materiales que permitan mejorar su funcionalidad en servicio.

\section{- Perfecto para la personalización} masiva: su flexibilidad para la personalización, la hacen óptima para lotes de producción pequeños de componentes y piezas de alto valor agregado.
- Menor desperdicio: permite el uso eficiente de materiales hasta en un $90 \%$ en la mayoría de los casos, es necesario observar la tecnología y el material a utilizar para evaluar su eficiencia.

\section{- Cadena de suministro más corta:} reduce la cantidad de proveedores al hacer posible que la producción se realice en sitio de baja demanda, interacción directa entre cliente y fabricante.

- Nuevos modelos comerciales: partes o productos completos pueden construirse bajo demanda, cuando y donde es necesario, teniendo enormes implicaciones sobre cómo los fabricantes diseñan, construyen y venden sus productos, se reduce necesidad de transporte, almacenamiento y costos de servicios.

Con el uso de la manufactura aditiva (MA) es posible diseñar y fabricar prótesis específicas y hechas a la medida para cada paciente, las cuales se adaptan a una anatomía única, lo que promete mejores resultados, corrigiendo de manera más confiable los problemas de alineación dentro de la rodilla gracias a las guías de corte diseñadas al mismo tiempo que la prótesis, y también evitando la eliminación innecesaria de hueso sano (Figura 8) [6, 39]. Asimismo, el desarrollo de la MA ha permitido el diseño y fabricación de armazones porosos cuyas aplicaciones pueden ser como alternativa al uso de 
cementos acrílicos para la fijación de las prótesis (osteointegración) [97] así como la adaptación de las propiedades mecánicas de las aleaciones utilizadas para la manufactura de estos componentes con valores más afines a los valores del hueso cortical y trabecular $[98,99]$.

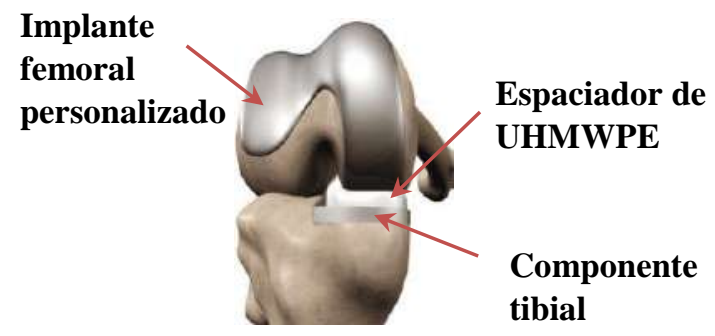

Figura 8. Prótesis de rodilla de Conformis diseñada a medida para un paciente específico [39]

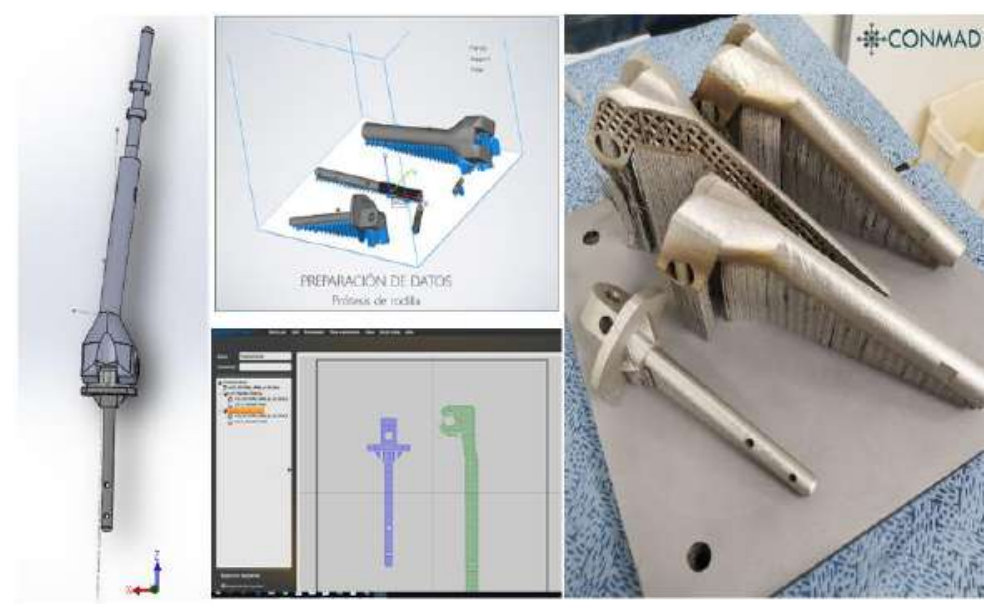

Figura 9. Proyecto de diseño y fabricación de prótesis de rodilla para niño por MA en el Consorcio CONACYT en Manufactura Aditiva (CONMAD).

\subsection{El futuro de los implantes ortopédicos en México}

En cuanto al estado actual en México, se puede ver que las instituciones públicas y privadas tienden, en su mayoría, a utilizar implantes de origen estadounidense $o$ europeo. Los criterios de esta selección son principalmente la calidad y la responsabilidad que conlleva el médico en este proceso.
La Figura 9 muestra algunos prototipos de componentes femorales y tibiales para prótesis de niño que fueron impresos en acero inoxidable usando la tecnología de fusión de cama de polvos asistida por láser. Sin embargo, a pesar del potencial y del interés encontrado en la MA de materiales para su aplicación en implantes, todavía hay retos a vencer, principalmente los costos elevados de inversión de la tecnología, la optimización de los procesos de manufactura para lograr implantes con propiedades confiables, reproducibles y biomiméticas con el tejido óseo circundante que busca reemplazar, así como de la materia prima que se utiliza y que en algunos casos debe ser importada, además de las consideraciones técnicas que han sido emitidas por las agencias reguladoras, p.ej., FDA [95]. 
rodilla o cadera para niños) se fabrican y/o se personalizan en el país en años recientes. En otros casos algunos implantes son diseñados en México y se manufacturan en el extranjero, incluyendo aquellos fabricados por MA.

Recientemente, el Consorcio CONACYT en manufactura aditiva (CONMAD, http://lisma.com.mx/), ha enfocado sus esfuerzos en el desarrollo de líneas de investigación orientadas al diseño, manufactura y protección de superficies de implantes metálicos y cerámicos, a través de la colaboración multiinstitucional y multidisciplinaria de centros de investigación, hospitales y empresas del sector médico. Uno de estos esfuerzos, es el diseño y fabricación de una prótesis de cadera para adultos mediante MA (ver Figura 10).

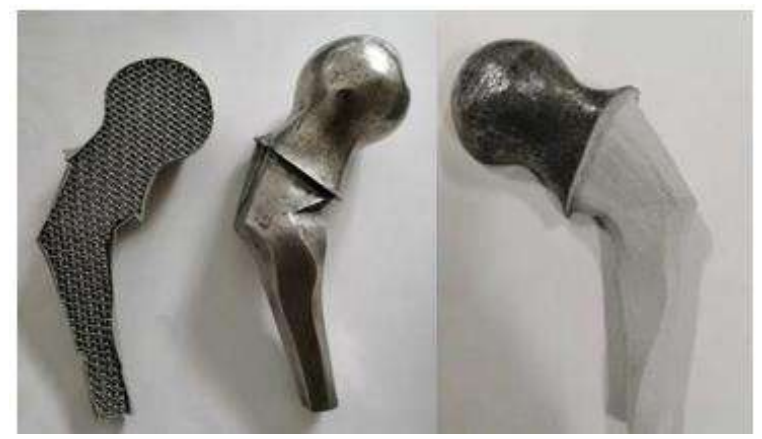

Figura 10. Prototipo impreso mediante impresión $3 D$ para cadera (medio) y recubierto con cerámico bioactivo mediante proyección térmica (derecha), fuente: Dirección de Materiales Avanzados CIATEQ A.C.

La prótesis en desarrollo, busca incluir estructuras celulares periódicas que le confieran propiedades mecánicas similares al hueso sin comprometer su desempeño en servicio. De esta forma, el módulo de Young del componente sería más afín al del hueso, mejorando la transferencia de esfuerzos y evitando la reabsorción ósea. Este es un desarrollo liderado por el CIATEQ A.C. en conjunto con instituciones médicas nacionales. A pesar de estos ejemplos y otros desarrollados en el país, los esfuerzos aún son incipientes y brindan la oportunidad de continuar investigando y mejorando los materiales utilizados en la fabricación de implantes, además de desarrollar las metodologías óptimas para la manufactura de implantes y los procesos para la conceptualización y diseño hechas en México. En este camino el trabajo colaborativo e interdisciplinario entre la academia, la industria, el gobierno y la sociedad, son inevitables. Nuevos conceptos de trabajo y proyectos de desarrollo tecnológico nacional que integren las capacidades de diseño, creadores de software y los centros de investigación con sus departamentos de manufactura y sus procesos de maquinado convencional e impresión 3D serán también necesarios. A su vez, todos estos enfoques deberán moverse bajo la sombra de las regulaciones internacionales de calidad de materiales y sistemas (ISO, ASTM), así como de los controles sanitarios mexicanos de la COFEPRIS (Comisión Federal para la Prevención de Riesgos Sanitarios).

\section{CONCLUSIONES}

En el mundo y en México, existe un fuerte problema de salud asociado a trastornos musculo-esqueléticos que causan lesiones severas en las articulaciones de la rodilla y cadera, lo que conlleva a la necesidad de colocar implantes ortopédicos. Además, se espera un incremento en el uso de estos reemplazos articulares debido a padecimientos que afectan a niños, jóvenes, y adultos.

Aunque los primeros resultados de la ATC y ATR fueron decepcionantes, se han producido avances constantes en las últimas décadas. Estos avances han permitido tanto el perfeccionamiento de la técnica quirúrgica como el desarrollo tecnológico con respecto al diseño de los dispositivos y los materiales para la fabricación de prótesis con el fin de proporcionar una técnica reproducible que 
ofrezca un buen rango de movimiento, estabilidad y, lo más importante, una vida útil adecuada. A medida que disminuya la edad promedio de quienes se someten a artroplastia de cadera y rodilla, estas consideraciones seguirán siendo de gran valor para aumentar la longevidad del implante en pacientes muy activos. Actualmente, la ATC y la ATR son cirugías que han marcado la historia médica moderna y los resultados clínicos de los últimos 30 años han demostrado que la ATC y la ATR son técnicas quirúrgicas exitosas para el tratamiento de afecciones degenerativas. El futuro de la ATC y la ATR debe centrarse no solo en la mejora de la función física, sino también en la calidad de vida del paciente, lo que conlleva a cambios en el diseño de prótesis a nivel geométrico, estructural, acabado superficial, y uso de mejores materiales.

Los retos identificados en los materiales utilizados para implantes ortopédicos están asociados al desgaste y corrosión que sufren en el cuerpo. Por lo tanto, es necesario la mejora de la resistencia a la abrasión de cementos óseos, así como su modificación con elementos bioactivos como la hidroxiapatita para que sea visible a los rayos $X$. Asimismo, se requiere mejorar la resistencia al desgaste y al envejecimiento por oxidación del polietileno de ultra-alto peso molecular utilizado en los reemplazos articulares. La mejora de los materiales poliméricos con aditivos base carbón (grafito, nanotubos, grafeno, etc) que son lubricantes sólidos naturales, así como el desarrollo de superficies auto-lubricantes como recubrimientos tipo diamante (DLC) o carburos parecen ser una alternativa prometedora, ya que además de reducir el desgaste del espaciador, los nanotubos, en específico, también ayudan a combatir el envejecimiento por oxidación del UHMWPE. Con esto se podrían reducir los casos de osteólisis y aflojamiento aséptico de los implantes causados por las partículas (Debris) de polietileno. Por su parte, la liberación de iones causada por la corrosión a largo plazo de los componentes de las aleaciones metálicas de los implantes debe ser seriamente considerada y evaluada.

A nivel internacional, la tendencia hacia la personalización de prótesis permitirá evaluar si la adecuación de las prótesis a la anatomía de cada paciente les da una mayor vida operativa y mejora en su función. Dentro del ámbito de la personalización de prótesis, un gran reto a abordar será la adaptación del módulo elástico de los materiales metálicos utilizados para la fabricación de implantes (componentes femorales y tibiales de la prótesis total de rodilla $\mathrm{y}$ vástago $\mathrm{y}$ componente acetabular de la prótesis total de cadera y ATC), para que sea más afín al módulo elástico del hueso cortical y trabecular. Asimismo, la manufactura aditiva (impresión 3D) podría convertirse en la tecnología clave para re-diseñar las prótesis que son completamente densas, migrándolas hacia sistemas de estructuras celulares 0 trabeculares.

En el simposio prótesis ortopédicas: Estatus actual en México https://protesismexico.com/ se pudo ver que existen diversos dispositivos de materiales metálicos y poliméricos ampliamente aceptados, certificados y autorizados para su aplicación en implantes y prótesis ortopédicas. Asimismo, se pudo ver que, a excepción de algunos desarrollos, la mayoría de las prótesis ortopédicas son importadas, y uno de los retos más grandes es el desarrollo de prótesis diseñadas para la anatomía del mexicano que cumplan con los estándares internacionales de calidad (ISO y ASTM) además de cumplir con las regulaciones sanitarias del país. Esto a su vez debe realizarse considerando la opinión clínica de médicos traumatólogos y ortopedistas. 


\section{AGRADECIMIENTOS}

Los autores agradecen el apoyo otorgado a través de los proyectos FORDECYT 297265 y 296384, y el proyecto 2015-02-1077 Programa Fronteras de la Ciencia administrado por CONACYT, y al Laboratorio Nacional CENAPROT- (sede CIDESI).

N. Camacho, A. Gómez-Ortega y G.C. Mondragón-Rodríguez agradecen el apoyo del CONACYT a través de su programa Cátedras CONACYT.

\section{REFERENCIAS BIBLIOGRÁFICAS}

[1] R. Vaishya and H. Lal, "Challenges, controversies, and innovations in arthroplasty," J Clin Orthop Trauma, vol. 9, no. 1, pp. 1-2, Jan. 2018.

[2] "Website." "Global RA Network " About Arthritis and RA." https://globalranetwork.org/project/diseaseinfo/\#: :text=Approximately $\% 2 \mathrm{C} \% 20$ one $\%$ 20per\%20cent\%20of,million\%20people\%20 live\%20with\%20RA. (accessed Sep. 30, 2020).

[3]http://www.imss.gob.mx/sites/all/statics/g uiasclinicas/435GER.pdf (accessed Jul. 31, 2019).

[4] "CIFRAS EN MÉXICO." https://amepar.org.mx/cifras-en-mexico/ (accessed Jul. 31, 2019).

[5]http://www.cenetec.salud.gob.mx/descarg as/gpc/CatalogoMaestro/435_GPC_Artropla stiaderodilla/435GER.pdf (accessed Jul. 31, 2019).

[6]http://www.larkinhospital.com/larkinorth opedics/wpcontent/uploads/2014/01/Artroplastia-Total-
de-Rodilla-20081.pdf (accessed Jul. 31, 2019).

[7] "Total Knee Replacement Statistics 2017: Younger Patients Driving Growth," Jul. 18, 2018. https://idataresearch.com/totalknee-replacement-statistics-2017-youngerpatients-driving-growth/ (accessed Sep. 27, 2020).

[8] "Cubos dinámicos." http://www.dgis.salud.gob.mx/contenidos/ba sesdedatos/BD_Cubos_gobmx.html (accessed Sep. 30, 2020).

[9] S. Trainor, J. Collins, H. Mulvey, and W. Fitz, "Total Knee Replacement Sizing: Shoe Size Is a Better Predictor for Implant Size than Body Height," Arch Bone Jt Surg, vol. 6, no. 2, pp. 100-104, Mar. 2018.

[10] B. Ravi, R. Croxford, W. M. Reichmann, E. Losina, J. N. Katz, and G. A. Hawker, "The changing demographics of total joint arthroplasty recipients in the United States and Ontario from 2001 to 2007," Best Practice \& Research Clinical Rheumatology, vol. 26, no. 5. pp. 637-647, 2012, doi: 10.1016/j.berh.2012.07.014.

[11] S. M. Kurtz et al., "International survey of primary and revision total knee replacement," Int. Orthop., vol. 35, no. 12, pp. 1783-1789, Dec. 2011.

[12] J. Baena, J. Wu, and Z. Peng, "Wear Performance of UHMWPE and Reinforced UHMWPE Composites in Arthroplasty Applications: A Review," Lubricants, vol. 3, no. 2. pp. 413-436, 2015, doi: 10.3390/lubricants3020413.

[13] A. M. Kandahari, X. Yang, K. A. Laroche, A. S. Dighe, D. Pan, and Q. Cui, " $A$ review of UHMWPE wear-induced osteolysis: the role for early detection of the immune response," Bone Research, vol. 4, no. 


\section{2016, doi: 10.1038/boneres.2016.14.}

[14] M. Nine, D. Choudhury, A. Hee, R. Mootanah, and N. Osman, "Wear Debris Characterization and Corresponding Biological Response: Artificial Hip and Knee Joints," Materials, vol. 7, no. 2. pp. 9801016, 2014, doi: 10.3390/ma7020980.

[15] M. C. S. Inacio, E. W. Paxton, S. E. Graves, R. S. Namba, and S. Nemes, "Projected increase in total knee arthroplasty in the United States - an alternative projection model," Osteoarthritis and Cartilage, vol. 25, no. 11. pp. 1797-1803, 2017, doi: 10.1016/j.joca.2017.07.022.

[16] L. Leitner et al., "Trends and Economic Impact of Hip and Knee Arthroplasty in Central Europe: Findings from the Austrian National Database," Sci. Rep., vol. 8, no. 1, p. 4707, Mar. 2018.

[17] F. Xie et al., "Evaluation of health outcomes in osteoarthritis patients after total knee replacement: a two-year follow-up," Health and Quality of Life Outcomes, vol. 8, no. 1 . p. 87 , 2010, doi: $10.1186 / 1477-7525-8$ 87.

[18] J. T. Evans, R. W. Walker, J. P. Evans, A. W. Blom, A. Sayers, and M. R. Whitehouse, "How long does a knee replacement last? A systematic review and meta-analysis of case series and national registry reports with more than 15 years of follow-up," Lancet, vol. 393, no. 10172, pp. 655-663, Feb. 2019.

[19] "Artificial Joint Replacement of the Knee | eOrthopod.com," eOrthopod.com, Jul. 08, 2015. https://eorthopod.com/artificialjoint-replacement-of-the-knee/ (accessed Jul. 31, 2019).

[20] "Reemplazo Total de Articulación de Rodilla." https://www.laorthocenter.com/espanol/educ ation.stryker_brochures.spanish.total_knee.3 .php (accessed Oct. 01, 2020).

[21] "Reemplazo Total de Rodilla." http://www.caderayrodilla.com.ar/reemplazo rodilla.htm (accessed Oct. 01, 2020).

[22] “Website.” A. A. o. H. a. K. Surgeon, «Hip and Knee Care Library,» American Association of Hip and Knee Surgeon, 2018. [En línea]. Available: https://hipknee.aahks.org/what-are-hip-andknee-replacement-implants-made-of/. [Último acceso: 1701 2019]. (accessed Jul. 31, 2019).

[23] "JOURNEY II XR Bi-Cruciate Retaining Knee System.” https://www.smithnephew.com/professional/products/orthopae dic-reconstruction/knee-

reconstruction/partial-and-total-knee/totalknee-replacement-portfolio/journey-ii-xr/ (accessed Nov. 09, 2020).

[24] B. Davies, K. L. Fan, R. D. Hibberd, M. Jakopec, and S. J. Harris, "A mechatronic based robotic system for knee surgery," Proceedings Intelligent Information Systems. IIS'97. doi: 10.1109/iis.1997.645177.

[25] W. M. Mihalko, "Additive Manufacturing of Arthroplasty Implants," 3D Printing in Orthopaedic Surgery. pp. 49-53, 2019, doi: 10.1016/b978-0-323-581189.00005-1.

[26] S. P. Narra, P. N. Mittwede, S. DeVincent Wolf, and K. L. Urish, "Additive Manufacturing in Total Joint Arthroplasty," Orthop. Clin. North Am., vol. 50, no. 1, pp. 13-20, Jan. 2019.

[27] W. Fitz, "Unicompartmental knee arthroplasty with use of novel patient-specific resurfacing implants and personalized jigs," J. Bone Joint Surg. Am., vol. 91 Suppl 1, pp. 
69-76, Feb. 2009.

[28] P. Jemmett and S. Roy, "The iDuo Bicompartmental Knee Replacement: Our Early Experience," Reconstructive Review, vol. 6, no. 4. 2016, doi: 10.15438/rr.6.4.149.

[29] M. Roche, K. A. Gustke, and T. Y. Law, "The utilization of smart trials in PCL retaining knees," Seminars in Arthroplasty, vol. 26, no. 4. pp. 218-228, 2015, doi: 10.1053/j.sart.2016.06.004.

[30] R. L. Barrack, T. Schrader, A. J. Bertot, M. W. Wolfe, and L. Myers, "Component rotation and anterior knee pain after total knee arthroplasty," Clin. Orthop. Relat. Res., no. 392, pp. 46-55, Nov. 2001.

[31] W. C. Schroer et al., "Why are total knees failing today? Etiology of total knee revision in 2010 and 2011," J. Arthroplasty, vol. 28, no. 8 Suppl, pp. 116-119, Sep. 2013.

[32] A. Postler, C. Lützner, F. Beyer, E. Tille, and J. Lützner, "Analysis of Total Knee Arthroplasty revision causes," BMC Musculoskeletal Disorders, vol. 19, no. 1. 2018, doi: 10.1186/s12891-018-1977-y.

[33] D. A. Camarata, "Soft tissue balance in total knee arthroplasty with a force sensor," Orthop. Clin. North Am., vol. 45, no. 2, pp. 175-184, Apr. 2014.

[34] "Website." Gustke K. Use of smart trials for soft-tissue balancing in total knee replacement surgery. J Bone Joint Surg $\mathrm{Br}$ 2012;94(11 Suppl A):147-50 (15) (PDF) Smart Sensor Technology to determine Softtissue Balance in Total Knee Arthroplasty. Available from: https://www.researchgate.net/publication/26 4979161_Smart_Sensor_Technology_to_det ermine_Soft-

tissue_Balance_in_Total_Knee_Arthroplasty [accessed Sep 11 2020]. (accessed Sep. 11,
2020).

[35] "Total Hip Replacement - OrthoInfo AAOS."

https://www.orthoinfo.org/en/treatment/total -hip-replacement/ (accessed Sep. 30, 2020).

[36] S. R. Knight, R. Aujla, and S. P. Biswas, "Total Hip Arthroplasty - over 100 years of operative history," Orthop. Rev. , vol. 3, no. 2, p. e16, Sep. 2011.

[37] A. Aherwar, A. K Singh, A. Patnaik, and Department of Mechanical Engineering, Malaviya National Institute of Technology, Malaviya Nagar, JLN Marg, Jaipur, Rajasthan-302017, India, "Current and future biocompatibility aspects of biomaterials for hip prosthesis," AIMS Bioengineering, vol. 3, no. 1, pp. 23-43, 2015.

[38] T.-K. Jung, H.-S. Lee, S. Semboshi, N. Masahashi, T. Abumiya, and S. Hanada, "A new concept of hip joint stem and its fabrication using metastable TiNbSn alloy," Journal of Alloys and Compounds, vol. 536. pp. S582-S585, 2012, doi: 10.1016/j.jallcom.2011.12.077.

[39] “Website." Conformis, «Conformis,» Conformis, 2019. [En línea]. Available: https://www.conformis.com/custom-madeknee-implants/. (accessed Jul. 31, 2019).

[40] M. Merola and S. Affatato, "Materials for Hip Prostheses: A Review of Wear and Loading Considerations," Materials , vol. 12, no. 3, Feb. 2019, doi: 10.3390/ma12030495.

[41] Z. You and D. Li, "The dynamical viscoelasticity and tensile property of new highly filled charcoal powder/ultra-high molecular weight polyethylene composites," Materials Letters, vol. 112. pp. 197-199, 2013, doi: 10.1016/j.matlet.2013.09.013. 
[42] S. Affatato, A. Ruggiero, and M. Merola, "Advanced biomaterials in hip joint arthroplasty. A review on polymer and ceramics composites as alternative bearings," Composites Part B: Engineering, vol. 83. pp. 276-283, 2015, doi: 10.1016/j.compositesb.2015.07.019.

[43] E. M. B. del Prever, E. M. B. del Prever, A. Bistolfi, P. Bracco, and L. Costa, "UHMWPE for arthroplasty: past or future," Journal of Orthopaedics and Traumatology, vol. 10, no. 1. pp. 1-8, 2009, doi: 10.1007/s10195-008-0038-y.

[44] J. S. Bergström, C. M. Rimnac, and S. M. Kurtz, "Prediction of multiaxial mechanical behavior for conventional and highly crosslinked UHMWPE using a hybrid constitutive model," Biomaterials, vol. 24, no. 8, pp. 1365-1380, Apr. 2003.

[45] R. Lerf, D. Zurbrügg, and D. Delfosse, "Use of vitamin E to protect crosslinked UHMWPE from oxidation," Biomaterials, vol. 31, no. 13, pp. 3643-3648, May 2010.

[46] E. Oral, C. Godleski Beckos, A. S. Malhi, and O. K. Muratoglu, "The effects of high dose irradiation on the cross-linking of vitamin E-blended ultrahigh molecular weight polyethylene," Biomaterials, vol. 29, no. 26, pp. 3557-3560, Sep. 2008.

[47] E. Oral, A. Neils, and O. K. Muratoglu, "High vitamin E content, impact resistant UHMWPE blend without loss of wear resistance," Journal of Biomedical Materials Research Part B: Applied Biomaterials, vol. 103, no. 4. pp. 790-797, 2015, doi: 10.1002/jbm.b.33256.

[48]https://www.cortical.com.br/catalogos/7 afd4b11fe4d5e372704e41398a9df13.pdf (accessed Nov. 08, 2020). [49]https://www.ceramtec.com/files/mt_biol ox_forte_delta_comparison_en.pdf (accessed Nov. 08, 2020).

[50] C. Piconi and A. A. Porporati, "Bioinert Ceramics: Zirconia and Alumina," Handbook of Bioceramics and Biocomposites. pp. 1-25, 2015, doi: 10.1007/978-3-319-09230-0_4-1.

[51] I. C. Clarke, D. D. Green, G. Pezzoti, and D. Donaldson, "20 Year Experience of Zirconia Total Hip Replacements," Ceramics in Orthopaedics. pp. 67-78, doi: 10.1007/37985-1540-9_12.

[52] S. Deville et al., "Low-temperature ageing of zirconia-toughened alumina ceramics and its implication in biomedical implants," J. Eur. Ceram. Soc., vol. 23, no. 15, pp. 2975-2982, Jan. 2003.

[53] M. D. Kohn, A. A. Sassoon, and N. D. Fernando, "Classifications in Brief: KellgrenLawrence Classification of Osteoarthritis," Clinical Orthopaedics and Related Research®, vol. 474, no. 8. pp. 1886-1893, 2016, doi: 10.1007/s11999-016-4732-4.

[54] S. de Salud, “Osteosarcoma, cáncer más frecuente en niños y adolescentes." http://www.gob.mx/salud/prensa/osteosarco ma-cancer-mas-frecuente-en-ninos-yadolescentes (accessed Sep. 28, 2020).

[55] N. D. Heckmann, L. Sivasundaram, M. D. Stefl, H. P. Kang, E. T. Basler, and J. R. Lieberman, "Total Hip Arthroplasty Bearing Surface Trends in the United States From 2007 to 2014: The Rise of Ceramic on Polyethylene," The Journal of Arthroplasty, vol. 33, no. 6. pp. 1757-1763.e1, 2018, doi: 10.1016/j.arth.2017.12.040.

[56] C. Rivière, C. Harman, T. Parsons, L. Villet, J. Cobb, and C. Maillot, "Kinematic alignment versus conventional techniques for 
total hip arthroplasty: A retrospective case control study," Orthop. Traumatol. Surg. Res., vol. 105, no. 5, pp. 895-905, Sep. 2019.

[57] D. H. Sochart and M. L. Porter, "The long-term results of Charnley low-friction arthroplasty in young patients who have congenital dislocation, degenerative osteoarthrosis, or rheumatoid arthritis," J. Bone Joint Surg. Am., vol. 79, no. 11, pp. 1599-1617, Nov. 1997.

[58] W. Y. Shon, B.-Y. Park, R. N. R, P. S. Park, J. T. Im, and H. H. Yun, "Total Hip Arthroplasty: Past, Present, and Future. What Has Been Achieved?," Hip Pelvis, vol. 31, no. 4, pp. 179-189, Dec. 2019.

[59] W. Y. Shon, T. Baldini, M. G. Peterson, T. M. Wright, and E. A. Salvati, "Impingement in total hip arthroplasty a study of retrieved acetabular components," J. Arthroplasty, vol. 20, no. 4, pp. 427-435, Jun. 2005.

[60] I. D. Learmonth, C. Young, and C. Rorabeck, "The operation of the century: total hip replacement," The Lancet, vol. 370, no. 9597. pp. 1508-1519, 2007, doi: 10.1016/s0140-6736(07)60457-7.

[61]https://cdigital.uv.mx/bitstream/handle/1 944/49969/FernandezArteagaAngel.pdf?seq uence $=1 \&$ isAllowed $=y$ (accessed Nov. 08, 2020).

[62] https://www.medigraphic.com/pdfs/inge/ ib-2001/ib012d.pdf (accessed Nov. 08, 2020).

[63] I.-G. Kang, C.-I. Park, H. Lee, H.-E. Kim, and S.-M. Lee, "Hydroxyapatite Microspheres as an Additive to Enhance Radiopacity, Biocompatibility, and Osteoconductivity of Poly(methyl methacrylate) Bone Cement," Materials , vol. 11, no. 2, Feb. 2018, doi:

\subsection{0/ma11020258}

[64] L. Shi et al., "The improved biological performance of a novel low elastic modulus implant," PLoS One, vol. 8, no. 2, p. e55015, Feb. 2013.

[65] M. Niinomi, Y. Liu, M. Nakai, H. Liu, and $\mathrm{H}$. Li, "Biomedical titanium alloys with Young's moduli close to that of cortical bone," Regenerative Biomaterials, vol. 3, no. 3. pp. 173-185, 2016, doi: 10.1093/rb/rbw016.

[66] M. T. Mohammed, "Mechanical Properties of SLM-Titanium Materials for Biomedical Applications: A Review," Materials Today: Proceedings, vol. 5, no. 9. pp. 17906-17913, 2018, doi: 10.1016/j.matpr.2018.06.119.

[67] T. Kunii et al., "Improved Osseointegration of a TiNbSn Alloy with a Low Young's Modulus Treated with Anodic Oxidation," Scientific Reports, vol. 9, no. 1. 2019, doi: 10.1038/s41598-019-50581-7.

[68] H. Matsumoto, S. Watanabe, and S. Hanada, "Microstructures and mechanical properties of metastable $\beta$ TiNbSn alloys cold rolled and heat treated," Journal of Alloys and Compounds, vol. 439, no. 1-2. pp. 146155, 2007, doi: 10.1016/j.jallcom.2006.08.267.

[69] Q. Guo, Y. Zhan, H. Mo, and G. Zhang, "Aging response of the Ti-Nb system biomaterials with $\beta$-stabilizing elements," Materials \& Design, vol. 31, no. 10. pp. 4842-4846, 2010, doi: 10.1016/j.matdes.2010.05.047.

[70] Oh, S. T., Woo, K. D., Kim, J. H., \& Kwak, S. M., "The Effect of $A l$ and $V$ on Microstructure and Transformation of $\beta$ Phase during Solution Treatments of Cast Ti6Al-4V Alloy," J. Korean Inst. Met. Mater., 
vol. 55, no. 3, pp. 150-155, Mar. 2017.

[71] L. Zhang and L. Chen, "A Review on Biomedical Titanium Alloys: Recent Progress and Prospect," Advanced Engineering Materials, vol. 21, no. 4. p. 1801215, 2019, doi: 10.1002/adem.201801215.

[72] G. A. Clavijo-Mejía, J. A. HermannMuñoz, J. A. Rincón-López, H. Ageorges, and J. Muñoz-Saldaña, "Bovine-derived hydroxyapatite coatings deposited by highvelocity oxygen-fuel and atmospheric plasma spray processes: A comparative study," Surface and Coatings Technology, vol. 381. p. 125193, 2020, doi: 10.1016/j.surfcoat.2019.125193.

[73] J. A. Hermann-Muñoz et al., "Influence of $H V O F$ parameters on HAp coating generation: An integrated approach using process maps," Surface and Coatings Technology, vol. 358. pp. 299-307, 2019, doi: 10.1016/j.surfcoat.2018.11.029.

[74] J. Henao et al., "HVOF Hydroxyapatite/Titania-Graded Coatings: Microstructural, Mechanical, and In Vitro Characterization," Journal of Thermal Spray Technology, vol. 27, no. 8. pp. 1302-1321, 2018, doi: 10.1007/s11666-018-0811-2.

[75] S. F. E, F. E. S., L. Shi, Z. G. Guo, and W. M. Liu, "The recent progress of tribological biomaterials," Biosurface and Biotribology, vol. 1, no. 2. pp. 81-97, 2015, doi: 10.1016/j.bsbt.2015.06.002.

[76] C. Fabry, C. Zietz, R. Dammer, and R. Bader, "12 Patterns of Wear in Total Knee Replacement," The Unhappy Total Knee Replacement. pp. 135-145, 2015, doi: 10.1007/978-3-319-08099-4_13.

[77]https://pdfs.semanticscholar.org/cc0e/7a e4c4f6022ef1011014b4d9740e802fb8c3.pdf (accessed May 20, 2020).
[78] G. W. Stachowiak, "Friction and Wear of Polymers, Ceramics and Composites in Biomedical Applications," Advances in Composite Tribology. pp. 509-557, 1993, doi: 10.1016/b978-0-444-89079-5.50018-0.

[79] N. Camacho, E. A. Franco-Urquiza, and S. W. Stafford, "Wear performance of multiwalled carbon nanotube-reinforced ultra-high molecular weight polyethylene composite," Advances in Polymer Technology, vol. 37, no. 6. pp. 2261-2269, 2018, doi: 10.1002/adv.21885.

[80] A. Bhattacharyya, S. Chen, and M. Zhu, "Graphene reinforced ultra high molecular weight polyethylene with improved tensile strength and creep resistance properties," Express Polymer Letters, vol. 8, no. 2. pp. 74-84, 2014, doi: 10.3144/expresspolymlett.2014.10.

[81] N. Dalai and P. S. Rama Sreekanth, "Mechanical properties of graphene and nano-diamond reinforced ultra high molecular weight polyethylene," Materials Today: Proceedings, vol. 27. pp. 1013-1016, 2020, doi: 10.1016/j.matpr.2020.01.350.

[82] B. P. Chang, H. M. Akil, R. B. Nasir, and A. Khan, "Optimization on wear performance of UHMWPE composites using response surface methodology," Tribology International, vol. 88. pp. 252-262, 2015, doi: 10.1016/j.triboint.2015.03.028.

[83] T. Liu, A. Eyler, and W.-H. Zhong, "Simultaneous improvements in wear resistance and mechanical properties of UHMWPE nanocomposite fabricated via a facile approach," Materials Letters, vol. 177. pp. 17-20, 2016, doi: 10.1016/j.matlet.2016.04.072.

[84] D. Lahiri, F. Hec, M. Thiesse, A. Durygin, C. Zhang, and A. Agarwal, 
"Nanotribological behavior of graphene nanoplatelet reinforced ultra high molecular weight polyethylene composites," Tribology International, vol. 70. pp. 165-169, 2014, doi: 10.1016/j.triboint.2013.10.012.

[85] N. T. Dintcheva et al., "Multifunctional hindered amine light stabilizersfunctionalized carbon nanotubes for advanced ultra-high molecular weight Polyethylene-based nanocomposites," Composites Part B: Engineering, vol. 82. pp. 196-204, 2015, doi: 10.1016/j.compositesb.2015.07.017.

[86] M. J. Martínez-Morlanes, P. Castell, P. J. Alonso, M. T. Martinez, and J. A. Puértolas, "Multi-walled carbon nanotubes acting as free radical scavengers in gammairradiated ultrahigh molecular weight polyethylene composites," Carbon, vol. 50, no. 7. pp. 2442-2452, 2012, doi: 10.1016/j.carbon.2012.01.066.

[87] M. J. Martínez-Morlanes, P. Castell, V. Martínez-Nogués, M. T. Martinez, P. J. Alonso, and J. A. Puértolas, "Effects of gamma-irradiation on UHMWPE/MWNT nanocomposites," Composites Science and Technology, vol. 71, no. 3. pp. 282-288, 2011, doi: 10.1016/j.compscitech.2010.11.013.

[88] Y. Xue, W. Wu, O. Jacobs, and B. Schädel, "Tribological behaviour of UHMWPE/HDPE blends reinforced with multi-wall carbon nanotubes," Polymer Testing, vol. 25, no. 2. pp. 221-229, 2006, doi: 10.1016/j.polymertesting.2005.10.005.

[89] P. S. R. Sreekanth, P. S. Rama Sreekanth, and S. Kanagaraj, "Influence of multi walled carbon nanotubes reinforcement and gamma irradiation on the wear behaviour of UHMWPE," Wear, vol. 334335. pp. 82-90, 2015, doi: 10.1016/j.wear.2014.12.014.
[90] A. Fonseca, S. Kanagaraj, M. S. A. Oliveira, and J. A. O. Simões, "Enhanced UHMWPE Reinforced with MWCNT through Mechanical Ball-Milling," Defect and Diffusion Forum, vol. 312-315. pp. 12381243 , 2011 , doi: 10.4028/www.scientific.net/ddf.312315.1238 .

[91] P. S. Rama Sreekanth and S. Kanagaraj, "Assessment of bulk and surface properties of medical grade UHMWPE based nanocomposites using Nanoindentation and microtensile testing," J. Mech. Behav. Biomed. Mater., vol. 18, pp. 140-151, Feb. 2013.

[92] N. Camacho, J. D. EscobedoRodríguez, J. M. Alvarado-Orozco, and G. C. Mondragón-Rodríguez, "Effect of SelfLubricating Carbon Materials on the Tribological Performance of Ultra-HighMolecular-Weight Polyethylene," Sep. 22, 2020.

[93] M. Masini, A. Levine, and A. Cruz, "Radiographic and Clinical Outcomes in Total Hip Arthroplasty Utilizing a Porous Acetabular Shell Developed with Additive Manufacturing." doi: 10.29007/bhs9.

[94] Wohlers Report 2018: Additive Manufacturing and $3 D$ Printing State of the Industry: Annual Worldwide Progress Report. 2018.

[95] Center for Devices and Radiological Health, "Technical Considerations for Additive Manufactured Medical Devices," Feb. 03 , 2020. https://www.fda.gov/regulatoryinformation/search-fda-guidancedocuments/technical-considerationsadditive-manufactured-medical-devices (accessed Sep. 11, 2020). 
[96] A. Nazir, K. M. Abate, A. Kumar, and J.-Y. Jeng, "A state-of-the-art review on types, design, optimization, and additive manufacturing of cellular structures," The International Journal of Advanced Manufacturing Technology, vol. 104, no. 912. pp. 3489-3510, 2019, doi: 10.1007/s00170-019-04085-3.

[97] C.-C. Huang et al., "Novel design of additive manufactured hollow porous implants," Dent. Mater., Sep. 2020, doi: 10.1016/j.dental.2020.08.011.

[98] H. Attar, S. Ehtemam-Haghighi, N.
Soro, D. Kent, and M. S. Dargusch, "Additive manufacturing of low-cost porous titaniumbased composites for biomedical applications: Advantages, challenges and opinion for future development," Journal of Alloys and Compounds, vol. 827. p. 154263, 2020, doi: 10.1016/j.jallcom.2020.154263.

[99] L. Yuan, S. Ding, and C. Wen, "Additive manufacturing technology for porous metal implant applications and triple minimal surface structures: A review," Bioactive Materials, vol. 4. pp. 56-70, 2019, doi: 10.1016/j.bioactmat.2018.12.003. 